\title{
LA INTERNACIONALIZACIÓN UNIVERSITARIA DESDE UNA PERSPECTIVA SITUADA: TENSIONES Y DESAFÍOS PARA LA REGIÓN LATINOAMERICANA
}

\begin{abstract}
UNIVERSITY INTERNATIONAL PERSPECTIVE LOCATED: TENSIONS AND CHALLENGES FOR THE LATIN AMERICAN REGION

A INTERNACIONALIZAÇÃO DA UNIVERSIDADE A PARTIR DE UMA PERSPECTIVA SITUADA: TENSÕES E DESAFIOS PARA A REGIÃO LATINOAMERICANA

María Soledad Oregioni ${ }^{1}$

RESUMEN

El artículo pone en discusión el concepto internacionalización universitaria, desde una perspectiva de análisis histórica y contextual, situada en la Región latinoamericana. En primer lugar, se identifican los actores y agentes de internacionalización, los instrumentos que promueven, y las tensiones que emergen en la internacionalización de las diferentes dimensiones de la vida universitaria (investigación, docencia, extensión), con el objetivo de dar cuenta de la complejidad del fenómeno, su carácter no neutral y la multiplicidad de aspectos que lo constituyen. En este sentido, a partir del análisis bibliográfico y del estudio de casos, se identifican diferentes formas de internacionalización, qué en términos analíticos se describen como internacionalización hegemónica, internacionalización no hegemónica, y contra-hegemónica. Y, en segundo lugar, se presenta a las Redes regionales de producción de conocimiento, como un instrumento apropiado para la internacionalización universitaria integral, donde interactúan las diferentes funciones de la vida universitaria en la generación y transmisión de conocimiento pertinente, desde una perspectiva no-hegemónica. Por último, se arriba a las reflexiones finales, planteando que abordar la internacionalización universitaria en toda su complejidad, es fundamental, para arribar a una mirada holística del fenómeno que contribuya a la planificación política y la proyección institucional.
\end{abstract}

PALABRAS CLAVE: Internacionalización universitaria. Producción de conocimiento. América Latina. Endógeno. Exógeno. Hegemónico. Contra-hegemónico.

\section{RESUMO}

O artigo faz discussão o conceito internacionalização da universidade, a partir de uma perspectiva histórica e conceitual, localizada na América Latina. Em primeiro lugar, identifica-se: os atores e agentes de internacionalização, instrumentos que promovam e tensões emergentes na internacionalização das diferentes dimensões da vida universitária (pesquisa, ensino, extensão), a fim de explicar a complexidade do fenómeno, o seu carácter não é neutro e a multiplicidade de aspectos que a constituem. Nesse sentido, a partir da revisão de literatura e estudos de caso, identifica-se diferentes formas de internacionalização, o que em termos analíticos são descritos como internacionalização hegemónica, a internacionalização não-hegemónica e contra hegemônica. E em segundo lugar, apresenta-se às redes regionais de produção de conhecimento, como um instrumento adequado para a internacionalização da universidade abrangente, onde interagem as diferentes funções da vida universitária na geração e transmissão de conhecimento relevante, do ponto de vista nãohegemónicos. Por fim, até as reflexões finais, afirmando que o endereço internacionalização universidade em toda a sua complexidade, é essencial para se chegar a uma visão holística do fenômeno que contribui para o planejamento de políticas e de projeção institucional.

PALAVRAS-CHAVE: Internacionalização da universidade. Produção de conhecimento. América Latina. Endógeno. Exógeno. Hegemônica. Contra hegemônica.

\footnotetext{
${ }^{1}$ Consejo Nacional de Investigaciones Científicas y Técnicas (CONICET), Universidad Nacional del Centro de la Provincia de Buenos Aires, Argentina. E-mail: soregioni08@ hotmail.com - ORCID: http://orcid.org/00000002-5741-0428
}

Submetido em: 22-10-2016 / Aceito em: 12-12-2016 / Publicado em: 14-02-2016.

\begin{tabular}{|c|c|c|c|c|c|c|} 
(C) Rev. Inter. Educ. Sup. & Campinas, SP & v.3 & n.1 & p.114-133 & jan./abr. 2017 & ISSN 2446-9424 \\
\hline
\end{tabular}




\begin{abstract}
The article discusses the concept of university internationalization from a perspective of historical and contextual analysis, located in the Latin American Region. In the first place, the actors and agents of internationalization, the instruments they promote, and the tensions that emerge in the internationalization of the different dimensions of university life (research, teaching, extension) are identified, in order to account for the complexity of the phenomenon, its non-neutral character and the multiplicity of aspects that constitute it. In this sense, from the bibliographic analysis and the case study, different forms of internationalization are identified, which in analytical terms are described as hegemonic internationalization, non-hegemonic internationalization, and counter-hegemonic. And secondly, regional networks of knowledge production are presented as an appropriate instrument for integral university internationalization, where the different functions of university life interact in the generation and transmission of relevant knowledge, from a non-hegemonic perspective. Finally, it is up to the final reflections, stating that in order to approach university internationalization in all its complexity, it is fundamental, to arrive at a holistic view of the phenomenon that contributes to the political planning and the institutional projection.
\end{abstract}

KEYWORDS: University internationalization. Knowledge production. Latin America. Endogenous. Exogenous. Hegemonic. Counter-hegemonic.

\title{
INTRODUCCIÓN
}

La internacionalización de las universidades no es un fenómeno nuevo, aunque en los últimos años se ha convertido en objeto de debates y manifiesta una nueva dinámica institucional (Didou Aupetit, 2007). El artículo describe las particularidades que presenta la internacionalización universitaria en la Región latinoamericana, a partir de profundizar cómo incide sobre las actividades de: investigación; docencia, extensión y gestión, ya que la internacionalización es transversal a dichas dimensiones de la vida universitaria. Además se presenta desde una perspectiva situada, directamente relacionada con las características de las universidades latinoamericanas. En primer lugar, porque las universidades son los principales centros de producción de conocimiento de la Región, en este sentido, es fundamental estudiar la internacionalización de la investigación en la universidad. En segundo lugar, porque la tradición de las universidades latinoamericanas tiene como pilar las actividades de extensión, actividad que es contemplada en varios programas de internacionalización orientada principalmente hacia los países de la Región. En tercer lugar, la internacionalización de la docencia-enseñanza, que tradicionalmente se aborda desde los estudios de Educación Superior (ES) adquiere nuevas modalidades de orden cuantitativo y cualitativo, influenciadas por la globalización. Todas las actividades (investigación, docencia, y extensión) son abordadas desde la universidad, y sus principales actores son docentes-investigadores. Asimismo, en el desarrollo de la internacionalización universitaria, intervienen agentes y actores de orden institucional, nacional, regional e internacional, mediante actividades de gestión, promoción y evaluación.

Para ingresar en el estudio de la internacionalización universitaria en la Región latinoamericana es necesario tener en consideración su estado del arte, en el año 2014, la UNESCO presenta el libro Internacionalización de la Educación Superior y la Ciencia en América Latina y el Caribe: Un Estado del Arte. Coordinado por Sylvie Didou Aupetit y Vielka Jaramillo de Escobar, que permite conocer los diferentes abordajes que se han

\begin{tabular}{l|l|l|l|l|l|l|} 
(C) Rev. Inter. Educ. Sup. & Campinas, SP & v.3 & n.1 & p.114-133 & jan./abr. 2017 & ISSN 2446-9424 \\
\hline
\end{tabular}


presentado en la Región sobre este fenómeno de estudio. Por otro lado, en trabajos previos se clasifican distintas perspectivas de análisis, estas son: 'perspectiva contextual', que entiende a la internacionalización como una respuesta a los cambios de orden cuantitativo y cualitativo generados por la globalización a partir de: 1) la incidencia de las tecnologías de la información y la comunicación; 2) cambios curriculares; 3) incremento de las movilidades; 4) procesos de integración regional en ES y homologación de titulaciones, como el Mercosur Educativo y el proceso Bolonia ${ }^{2}$. En segundo lugar, la 'perspectiva institucional' enfatiza en un nuevo estilo de gestión y un cambio en la cultura institucional (Sebastián, 2004; Knight, 1999). Por último, la 'perspectiva crítica' reúne trabajos que dan cuenta de los aspectos negativos de la internacionalización de las universidades, a partir de identificar a la globalización y transnacionalización de las instituciones universitarias, con la mercantilización de los servicios educativos, y la permanencia de un esquema convencional de distribución espacial de los flujos de estudiantes y académicos en el extranjero desde el Sur hacia el Norte, denominado 'fuga de cerebros' (ALTBACH, 2004, GASCÓN; CEPEDA, 2004; BERNAL, 2007, YARZÁBAL, 2005). Las diferentes perspectivas ponderan las causas y consecuencias de la internacionalización de la universidad, no son excluyentes, e incluso pueden ser complementarias. (OREGIONI, 2013)

Desde una perspectiva histórica y contextual el artículo busca presentar elementos de análisis que permitan generar nuevos abordajes de la internacionalización universitaria a partir de las características que presentan las universidades latinoamericanas, y de esta forma transitar desde nuestras realidades hacia nuevas ideas, con el objetivo último de transformar la realidad, teniendo en cuenta la particularidad y la diversidad del panorama universitario en América latina.

En términos analíticos podemos distinguir entre distintos tipos de internacionalización: "exógena", "hegemónica", "endógena", "no hegemónica", e incluso "contra-hegemónica" esto en la práctica no se identifica en forma "pura", por el contrario, en las distintas universidades estos aspectos u orientaciones de la internacionalización se encuentran en constante tensión.

Cuadro 1. Elementos conceptuales

\begin{tabular}{|c|c|}
\hline $\begin{array}{c}\text { INTERNACIONALIZACIÓN } \\
\text { HEGEMÓNICA }\end{array}$ & $\begin{array}{c}\text { INTERNACIONALIZACIÓN } \\
\text { NO-HEGEMÓNICA }\end{array}$ \\
\hline $\begin{array}{c}\text { EXÓGENA } \\
\text { Incentivada por proyectos, actores, intereses } \\
\text { extra-regionales }\end{array}$ & $\begin{array}{c}\text { ENDÓGENA } \\
\text { La estrategia de internacionalización se elabora } \\
\text { acorde a la planificación de la universidad } \\
\text { orientada por organismos nacionales y regionales. }\end{array}$ \\
\hline
\end{tabular}

\footnotetext{
${ }^{2}$ Entre los autores que abordan la internacionalización de la Educación desde una perspectiva contextual podemos identificar a: Brunner, 1999; Rama, 2006; Gacel- Ávila, 2000; Fernández López y Ruzo Sanmartín, 2004; García Guadilla, 2003, 2005, 2010; Jaramillo y De Lisio, 2004; Rama, 2006; Donini, 2010. (Ver: OREGIONI, 2013)
} 


\begin{tabular}{|c|c|}
\hline MERCANTIL & SOLIDARIA \\
\hline $\begin{array}{l}\text { La internacionalización se genera con fines } \\
\text { de lucro. Prima la racionalidad económica. }\end{array}$ & $\begin{array}{l}\text { La internacionalización se construye a partir de } \\
\text { lazos de cooperación internacional. }\end{array}$ \\
\hline
\end{tabular}

Fuente: Elaboración propia (Oregioni, 2015)

Cómo se puede observar en el cuadro 1, a partir del análisis bibliográfico y del estudio de casos, se han identificado diferentes formas de internacionalización. En la definición de la internacionalización hegemónica, que se presenta en la región latinoamericana en general y mercosureña en particular, se contemplan dos elementos. En primer lugar, el carácter exógeno de los vínculos internacionales, ya que es incentivada por proyectos, actores e intereses extra regionales, que históricamente guiaron las políticas de vinculación asimétricas norte-sur. En segundo lugar, su lógica mercantilista ${ }^{3}$, en la medida que se encuentra guiada por los vínculos económicos e identifica a la ES como un servicio a ser comercializado.

Por otro lado, en la columna ubicada a la derecha del cuadro 1, se presenta a la internacionalización no hegemónica, caracterizada por proyectarse endógenamente, es decir que la estrategia de internacionalización se elabora acorde a la planificación de la universidad orientada por organismos nacionales y regionales. Y se basa en prácticas de internacionalización solidarias, a partir de vínculos de cooperación entre las universidades. Son varios los autores latinoamericanos que presentan el concepto de internacionalización solidaria, entre ellos podemos mencionar a Nairdof (2005); Rojas Mix (2005); Siufi (2009); dicha definición busca dar cuenta de los lazos que se generan en la búsqueda de beneficios mutuos y de co-construcción de conocimiento. La internacionalización no-hegemónica se transforma en contra-hegemónica en la medida que encuentra instrumentos para la acción en pos de fomentar características de internacionalización diferentes a las tendencias dominantes a nivel internacional en forma planificada. En este sentido, las redes de producción y difusión de conocimiento entre universidades de la Región, se constituyen en un instrumento de internacionalización contra-hegemónico (OREGIONI, et. al; 2014).

\section{UNA MIRADA TRANSVERSAL SOBRE LA INTERNACIONALIZACIÓN UNIVERSITARIA}

Desde el ámbito académico se coincide en señalar el carácter transversal de la internacionalización universitaria. La autora más referenciada es Knight (1994) quién desde una perspectiva institucional, plantea que internacionalización de la universidad "es el proceso de incorporar una dimensión internacional/intercultural en las funciones de la enseñanza, la investigación y el servicio de la institución” (KNIGHT, 1994, p. 2).

\footnotetext{
${ }^{3}$ Qué Perrotta (2015) identifica para el caso del MERCOSUR como internacionalización fenicia.
}

\begin{tabular}{|l|l|l|l|l|l|l|} 
(C) Rev. Inter. Educ. Sup. & Campinas, SP & v.3 & n.1 & p.114-133 & jan./abr. 2017 & ISSN 2446-9424 \\
\hline
\end{tabular}


En este sentido, el trabajo invita a pensar la internacionalización universitaria situada en la Región latinoamericana desde una perspectiva histórica y contextual, donde las funciones sustantivas de la universidad cobran características específicas, dado que se contemplan las relaciones de poder que se dan en el ámbito internacional, el lugar que ha tenido históricamente América latina; y la particularidad y diversidad del panorama universitario en la Región.

Consecuentemente, en una primera instancia, se procederá a de-construir el concepto internacionalización universitaria (en términos analíticos) para observar las tensiones que emergen en la internacionalización de las diferentes funciones de la vida universitaria y, en segundo lugar, mostraremos como confluyen, dichas funciones cuando se trabaja en redes de producción y difusión de conocimiento, y en proyectos de carácter interdisciplinar orientados a la solución de problemas. Dando lugar a la internacionalización integral de la vida universitaria. En este sentido se presenta a las redes como instrumentos que permiten orientar la internacionalización hacia la integración regional desde una perspectiva nohegemónica, endógena e integral (OREGIONI, et. al, 2014). A continuación, el cuadro 2 permite identificar los instrumentos que generan estímulos a la internacionalización en las diferentes dimensiones de la vida universitaria, las tensiones que se presentan y los actores que participan, teniendo en cuenta que de acuerdo a Sebastián (2011) los actores tienen una función ejecutora y lo agentes una función promotora.

Cuadro 2. Elementos transversales a la internacionalización universitaria

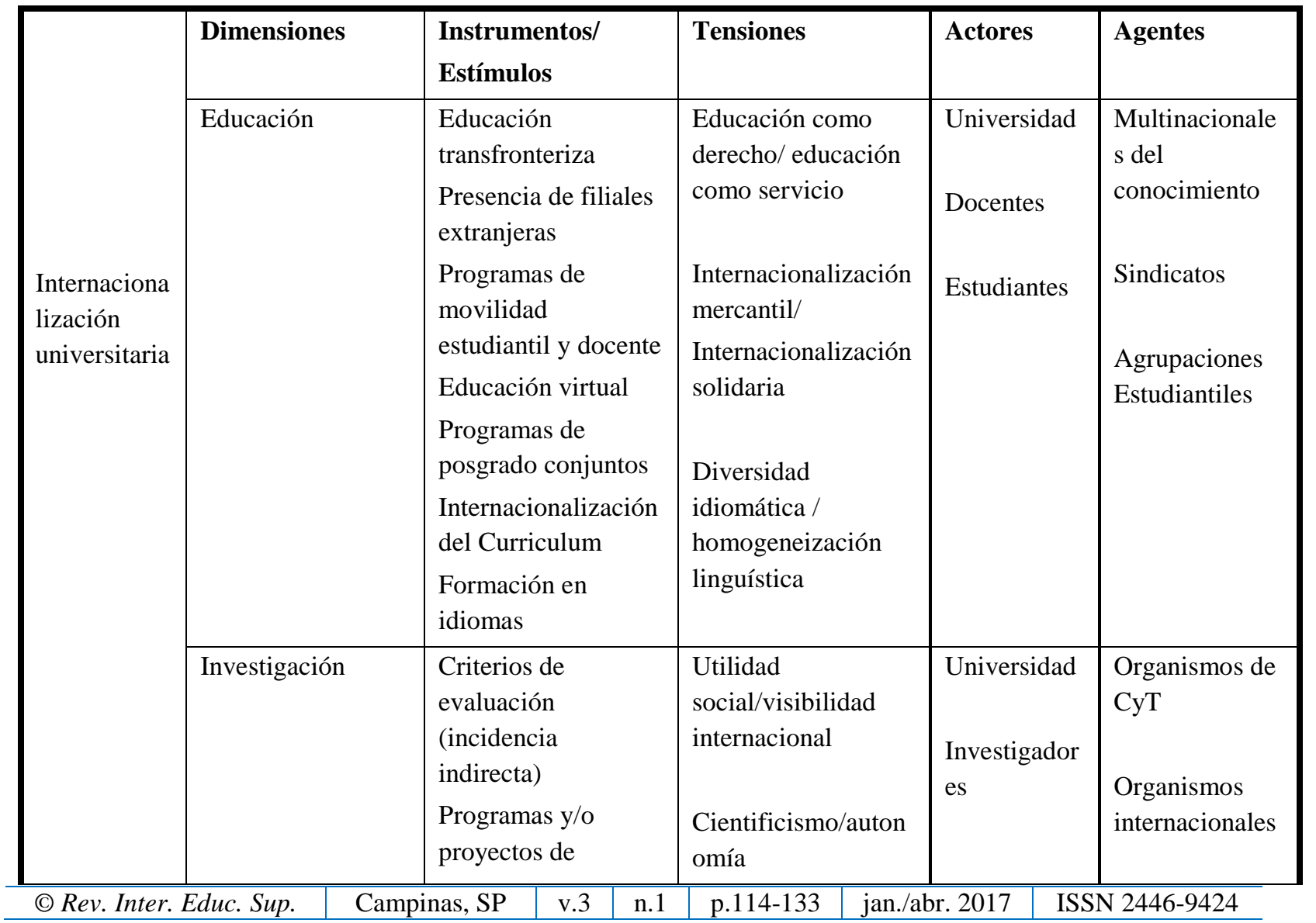




\begin{tabular}{|c|c|c|c|c|}
\hline & $\begin{array}{l}\text { cooperación } \\
\text { internacional } \\
\text { (incidencia directa) } \\
\text { Desarrollo de tesis } \\
\text { sándwich (co- } \\
\text { dirigidas) } \\
\text { Diásporas } \\
\text { científicas } \\
\text { Utilización de } \\
\text { equipamiento } \\
\text { conjunto } \\
\text { Redes de } \\
\text { producción de } \\
\text { conocimiento } \\
\text { Participación en } \\
\text { programas de } \\
\text { movilidad }\end{array}$ & $\begin{array}{l}\text { Legitimidad/eficien } \\
\text { cia-eficacia } \\
\text { Pensamiento } \\
\text { universal/pensamien } \\
\text { to situado }\end{array}$ & $\begin{array}{l}\text { Becarios } \\
\text { Pares de la } \\
\text { comunidad } \\
\text { científica. }\end{array}$ & $\begin{array}{l}\text { Programas } \\
\text { Regionales }\end{array}$ \\
\hline Extensión & $\begin{array}{l}\text { Programas de ayuda } \\
\text { humanitaria } \\
\text { Programas de } \\
\text { integración regional } \\
\text { Vinculación con } \\
\text { movimientos } \\
\text { sociales } \\
\text { Articulación con } \\
\text { pares no académicos } \\
\text { Divulgación } \\
\text { Talleres } \\
\text { multiculturales } \\
\text { Actividades de } \\
\text { voluntariado }\end{array}$ & $\begin{array}{l}\text { Proyectos } \\
\text { unidireccionales/ } \\
\text { Proyectos } \\
\text { interactivos }\end{array}$ & $\begin{array}{l}\text { Universidad } \\
\text { Extensionist } \\
\text { as } \\
\text { Empresas } \\
\text { Movimiento } \\
\text { s Sociales } \\
\text { Organizacio } \\
\text { nes de la } \\
\text { Sociedad } \\
\text { Civil }\end{array}$ & $\begin{array}{l}\text { Organismos } \\
\text { Regionales } \\
\text { Organismos } \\
\text { Internacionales } \\
\text { Organismos } \\
\text { gubernamental } \\
\text { es }\end{array}$ \\
\hline
\end{tabular}

Fuente: Elaboración propia.

En el cuadro 2 se destaca la transversalidad de la internacionalización respecto a las funciones sustantivas de la universidad, algunos de los puntos mencionados se han abordado en trabajos anteriores y otros se profundizarán en futuras investigaciones. El objetivo es dar cuenta de la complejidad del fenómeno, y la multiplicidad de aspectos que lo constituyen, esto no da lugar a abordajes simplistas o lineales del mismo, pero si requiere tener una mirada holística para la planificación política y proyección institucional.

En trabajos previos Jesús Sebastian $(2004,2007$, 2011) presenta las diferentes dimensiones de la internacionalización, los agentes y actores que intervienen en las universidades de la Región, que se han analizado puntualmente a partir de estudios de caso (OREGIONI, 2013, 2014). En este sentido, a partir de entender a la internacionalización universitaria como una actividad trasversal a los aspectos sustantivos de la vida universitaria, y por lo tanto que forman parte de las actividades cotidianas de docentes investigadores, nos interesa puntualizar en las tensiones que se presentan dado el carácter político de este

\begin{tabular}{l|c|c|c|c|c|c|} 
(C) Rev. Inter. Educ. Sup. & Campinas, SP & v.3 & n.1 & p.114-133 & jan./abr. 2017 & ISSN 2446-9424 \\
\hline
\end{tabular}


fenómeno, y la importancia que tienen en la orientación de la producción y transmisión de conocimiento. Por ejemplo, entran en tensión un modelo de internacionalización que presenta a la ES como servicio y un modelo qué presenta a la ES como derecho; el anclaje territorial en tensión con la orientación homogeneizarte; la generación de conocimiento competitivo en tensión respecto a la generación de conocimiento pertinente; medir la internacionalización a partir de la cantidad de convenios internacionales o de acuerdo a la calidad y pertinencia de los vínculos internacionales, etc. Asimismo, para tener un panorama general del fenómeno es necesario identificar la incidencia de discusiones, elementos, marcos regulatorios a nivel: regional, nacional e institucional.

\section{Tensión entre ES como servicio y ES como derecho}

En primer lugar, es importante aclarar que entendemos que la internacionalización de la ES forma parte de la internacionalización universitaria, aunque no son lo mismo, dado que la universidad incluye las funciones de investigación y extensión, y por lo tanto incluye discusiones que se dan en otros campos de producción de conocimiento, por ejemplo, el campo de los estudios sociales en ciencia y tecnología.

Partiendo desde una perspectiva contextual, tal como sostiene Yarzábal (2005), el siglo XXI es testigo de una orientación mercantil de la internacionalización de la ES, a partir de la conformación de un mercado mundial de ES que tiene como epicentro a los países angloparlantes. De esta forma, mientras que, en el transcurso de la década del noventa del siglo XX, la UNESCO coordinaba un debate altamente participativo en busca del cambio y el desarrollo de la ES que incluía la cooperación académica internacional como factor de progreso, los gestores de la "industria educativa" calibraban las características de los mercados y actuaban rápidamente ante su creciente y prometedora magnitud. (YARZÁBAL, 2005)

En este sentido, Altbach (2004) denuncia al GATS ${ }^{4}$ como un marco regulatorio que promueven las corporaciones multilaterales y los gobiernos interesados en exportar servicios educativos. En respuesta a esto en la Conferencia Regional sobre ES del año 2008, los Estados latinoamericanos han avanzado en identificar los riesgos que conlleva incorporar acríticamente los postulados de los organismos financieros internacionales, a partir de señalar que:

La incorporación de la Educación como un servicio comercial en el marco de la OMC ha dado lugar al rechazo generalizado por parte de muy diversas organizaciones relacionadas directamente con la Educación Superior (...) advertimos a los Estados de América Latina y Caribe sobre los peligros que implica aceptar los acuerdos de la OMC y luego estar obligados por estos, entre

\footnotetext{
${ }^{4}$ Acuerdo General sobre el Comercio de Servicios o AGCS (en inglés: General Agreement on Trade in Services o GATS).
} 
otros aspectos, a orientar fondos públicos hacia emprendimientos privados extranjeros implantados en su territorio, en cumplimiento del principio de "trato nacional" que en ellos se establece. Asimismo, afirmamos nuestro propósito de actuar para que la Educación en General, y la Educación Superior en particular no sean consideradas como servicio comercial" (...) "la educación suministrada por proveedores transnacionales, exenta de control y orientación por parte de los Estados Nacionales, favorece la educación descontextualizada en la cual los principios de pertinencia y equidad quedan desplazados. Ello amplia la exclusión, fomenta la desigualdad y consolida el subdesarrollo. (CRES, 2008)

En Argentina, a instancias de este evento que se incrementan las reflexionar académicamente sobre el fenómeno de la Internacionalización de la ES (FERNÁNDEZ LAMARRA, 2009). La crítica a la perspectiva hegemónica sobre la Internacionalización, se dirige a que se enfrenta a corrientes que promueven su mercantilización y privatización así como la falta de apoyo por parte del Estado que la concibe como un servicio y no como un derecho, la desregulación y deslocalización en la provisión de ES amenaza a los ideales tradicionales y al control institucional y nacional; es gobernada por la racionalidad económica e intereses externos; generando la pérdida de control por parte de las universidades.

Las voces críticas hacia la relación entre Internacionalización de la ES y su mercantilización se profundizan a partir de la incorporación de la ES al Acuerdo General sobre Comercio de Servicios de la OMC como un "bien" más a ser progresivamente liberalizado (GASCÓN; CEPEDA, 2004, YARZÁBAL, 2005). Este suceso sería la llave que abriría las puertas a la conformación de una 'industria educativa a nivel mundial' de la mano de actores externos (que en muchos casos tienen como único objetivo la ganancia) afectando negativamente a la calidad educativa, la pertinencia social de la educación y la democratización en el acceso. En este sentido, si se plantea a la ES como un bien público global, deja de estar sujeta a las normas, regulaciones y jurisprudencia de los estados nacionales y pasa a estar regulada por la Organización Mundial del Comercio.

Por otro lado, los efectos de la globalización a partir de la incorporación de las TIC constituyen uno de los instrumentos que favorecen la expansión de la educación transnacional, que constituye una de las aristas de la internacionalización universitaria. Ya que la educación virtual, hoy en pleno crecimiento, es global y transnacional por naturaleza (YAZABAL; 2005). De acuerdo con García Fanelli (1999, p. 88) la transnacionalización de la ES consiste en:

(...) cualquier actividad de enseñanza o aprendizaje en la cual los estudiantes están en un país diferente (el país huésped) de aquel al cual pertenece la institución proveedora (el país proveedor). Esta situación requiere que las fronteras nacionales sean cruzadas por información educativa y por los profesores y/o los materiales educativos. 
Según la autora, este fenómeno llevará a que la formación universitaria deje de ser privativa de una institución específica, ubicada en un único territorio y a través de la modalidad presencial. Esta transnacionalización universitaria, presenta diferentes formas de organización, entre las que identifica: 1) La instalación de campus foráneos por parte de universidades extranjeras, en el territorio nacional; 2) El desarrollo de alianzas universitarias que funcionan en torno a la provisión de una formación en alternancia o bien del otorgamiento del doble título, mediante acuerdos institucionales, modalidad que se encuentra en universidades públicas y privadas; 3) La consolidación de una oferta transnacional de licenciaturas y postgrados bajo la fórmula de la educación a distancia y virtual; 4) Arreglos entre instituciones para que una universidad privada sea huésped de una institución extranjera en un sistema de franquicia.

Ahora bien, esto conlleva al menos dos problemas a tener en cuenta: deslocalización del conocimiento y ausencia de regulaciones:

El primer problema es la deslocalización del conocimiento, es decir, que los conocimientos transmitidos en el "aula virtual" no se adecuen a las problemáticas, necesidades, e intereses de las poblaciones locales. Ya que la dinámica de transnacionalización de la ES manifiesta tensiones entre "conocimiento universal" y "saber situado", teniendo en cuenta el anclaje territorial de la universidad, y corrientes que promueven la desregulación y deslocalización de la provisión de ES, y amenaza los ideales tradicionales y el control regional, nacional e institucional, ya que es gobernada por la racionalidad económica de los proveedores externos. Esto implica la pérdida de control de las universidades sobre las actividades de docencia, de investigación y extensión, en tanto queda reducida en los acuerdos internacionales y las regulaciones de organismos internacionales.

El segundo problema identificado consiste en la ausencia de regulaciones, ya que proliferan fábricas de diplomas, que en la bibliografía especializada se identifica como "diploma mills", y hacen referencia a diplomas emitidos a alto consto y sin garantía de calidad. Por ejemplo, en el caso de EE.UU, con una creciente oferta de este tipo de actividades, las regulaciones de calidad no tienen la misma importancia en todos los estados (independientemente del concepto de calidad que manejen). En el caso de Nueva York por ejemplo, los título emitidos por Instituciones de ES no acreditadas, carecen de valor académico y profesional. En el caso de Europa, Reino Unido tiene una importante presencia en el mercado educativo transnacional y al igual que EE.UU es un centro de exportación, Francia y Holanda se encuentran más regulados y presentan escasa oferta de Instituciones de ES Privadas, y España, Grecia e Italia, constituyen centros de importación de programas educativos, principalmente provenientes de EE.UU y Reino Unido (GARCIA FANELLI, 1999). Los elementos mencionados, ponen en riesgo la concepción de la educación como un derecho que deben garantizar los Estados. 
Tensiones en la Internacionalización de la Investigación

La internacionalización de la investigación forma parte de la internacionalización universitaria, dado que las universidades públicas son los principales centros de producción de conocimiento en América latina, las investigaciones sobre este fenómeno provienen fundamentalmente del campo de los estudios sociales de la ciencia y la tecnología. En trabajos previos se ha identificado que la internacionalización atraviesa la dinámica de producción de conocimiento desde una perspectiva multidimensional, a nivel social, cognitivo e institucional, incidiendo en el contenido, la utilidad y la pertinencia del conocimiento que se genera en América latina (OREGIONI, 2014; 2015), ya que, las relaciones internacionales de los grupos de investigación locales con actores e instituciones del extranjero, se dan en un contexto internacional jerárquico caracterizado por asimetrías entre "centros" y "periferias" del conocimiento (VESSURI, 1996). La relación centroperiferia atraviesa la dinámica de producción de conocimiento, en la medida que el 'centro' se caracteriza por la concentración de recursos (materiales, cognitivos, humanos y simbólicos) destinados a la producción de conocimiento (VESSURI, 1984) y se convierten en un punto de referencia y objeto de imitación acrítica para los científicos de la periferia (HODARA, 2003).

De esta forma, se observa que las prácticas de internacionalización hegemónicas orientan el conocimiento hacia los problemas cognitivos planteados en países centrales, que poseen mayor cantidad de recursos (cognitivos, materiales, humanos, etc.) considerando el conocimiento como "neutral" y "universal", y con pocos márgenes de negociación de los problemas cognitivos sobre los que trabajan (OREGIONI, 2014). Asimismo, se identifican prácticas de internacionalización de la investigación no-hegemónicas que adquieren dinámicas particulares, donde los investigadores realizan estrategias que les permite desarrollar su poder de agencia.

El estudio de la internacionalización de la investigación en la Universidad Nacional de La Plata permite identificar la vinculación que existe entre aspectos relacionales, que dan cuenta de las relaciones de poder e influencia entre los centros y periferias del conocimiento, y los elementos que lo sustentan en el ámbito local: aspectos políticos institucionales y aspectos socio-cognitivos, que forman parte de los investigadores/unidades de investigación.

Los aspectos político-institucionales: son las herramientas que elaboran los organismos de ciência y la universidad, que contribuyen o no la internacionalización de la investigación, y fundamentalmente a reproducir o contrarrestar las tendencias hegemónicas. Al mismo tiempo, las unidades de investigación (espacio donde desarrollan su trabajo los investigadores), y los investigadores que forman parte de ellas, dan cuenta de diferentes estrategias de negociación de recursos para desarrollar sus investigaciones, sostenerse en el sistema, y obtener legitimación de sus pares a partir de posicionarse en el campo académico a nivel internacional. En este sentido, se observan relaciones de poder (dominación/subordinación) que median la posición de los investigadores en el campo

\begin{tabular}{|l|l|l|l|l|l|l|} 
(C) Rev. Inter. Educ. Sup. & Campinas, SP & v.3 & n.1 & p.114-133 & jan./abr. 2017 & ISSN 2446-9424 \\
\hline
\end{tabular}


disciplinar, las políticas institucionales que contrarrestan o profundizan las tendencias hegemónicas.

De esta forma los investigadores/ unidades de investigación, tienen un poder de agencia relativo, respecto a profundizar las tendencias dominantes o llevar adelante medidas no-hegemónicas/contra-hegemónicas. Esto se puede observar a partir de las tensiones que media la producción de conocimiento, entre ellas: visibilidad internacional en tensión con la utilidad social del conocimiento; científicos academicistas en tensión con científicos críticos; cientificismo en tensión con autonomía.

Los científicos latinoamericanos históricamente se han debatido entre "la voluntad de incorporación al sistema científico internacional y el deseo de llegar a tener una voz propia, autonomía en la definición de su perfil y su legitimación" (VESSURI, 1994, p. 72). Son varios los trabajos que dan cuenta de la tensión entre utilidad social y visibilidad internacional que atraviesa la producción de conocimientos a partir de la participación de científicos locales en las mega redes internacionales de producción de conocimiento (KREIMER; ZABALA, 2006, KREIMER; MEYER, 2008) generando lo que Kreimer y Thomas (2005) denominaron como 'conocimiento aplicable no aplicado' (CANA) dado que el conocimiento resultante de dichas interacciones es poco o nada aplicable a las demandas de contexto socio-productivo local (Dagnino, 2006). En este sentido, se destaca la vigencia del pensamiento latinoamericano en ciencia, tecnología y desarrollo (PLACTED) que ejerce una fuerte crítica sobre la investigación que sigue parámetros puramente 'cientificistas' sin arribar a soluciones concretas para las necesidades de las poblaciones de la Región latinoamericana.

Por otro lado, los estudios de caso demuestran que si bien en los últimos diez años en Argentina se han implementado políticas e instrumentos para incrementar los vínculos entre investigadores latinoamericanos (becas CONICET para estudiantes latinoamericanos; promoción de redes de producción de conocimiento como AUGM, o los instrumentos promovidos por la Secretaría de Políticas Universitarias). Los parámetros de evaluación y criterios de "excelencia", mantienen la percepción de la ciencia como una actividad "universal", y del conocimiento que se produce en los centros internacionales como "excelencia científica", esto incide en los criterios de "calidad", ya que los evaluadores son los mismos pares académicos, ejerciendo una "incidencia indirecta" sobre la orientación de la internacionalización de la investigación (OREGIONI, 2015) Además trae correlacionada una serie de elementos o dimensiones de la producción de conocimiento (agenda de investigación, técnicas de investigación, instrumentos de investigación) que desvinculan a la investigación del contexto local y la tornan dependiente de los centros internacionales de producción de conocimiento (OREGIONI, 2014).

Este debate no es nuevo, pero merece ser señalado dado que los criterios de "calidad" se continúan fijando en función de la comunidad científica internacional, por sobre las características sociales, culturales y económicas del contexto de investigación. Como se repite en el discurso de los investigadores: "nos miden por las publicaciones que realizamos",

\begin{tabular}{l|l|l|l|l|l|l} 
(C) Rev. Inter. Educ. Sup. & Campinas, SP & v.3 & n.1 & p.114-133 & jan./abr. 2017 & ISSN 2446-9424 \\
\hline
\end{tabular}


o "para publicar en determinada revista es necesario dominar determinada técnica" y para eso se necesita tal equipo, no disponible en el país y por lo tanto se debe optar por: comprarlo, adquirir servicios de consultoría, o incorporarse a redes de cooperación internacional para su utilización. Esta dinámica se reproduce principalmente en las unidades de investigación del área temática de 'Ciencias Exactas y Naturales' y de 'Ciencias biológicas y de la Salud', pero además los criterios internacionalizados de evaluación se han incorporado en el área temática de las Ciencias Sociales y de las Ingenierías y Ciencias de los Materiales. ${ }^{5}$ Por lo tanto, se otorga mayor puntaje a aquellos investigadores que publican en revistas reconocidas a nivel internacional, alterando los patrones originales de producción y divulgación de conocimiento propias del campo. Tal como advierte Pellegrino (2000), esto puede desincentivar el desarrollo del conocimiento en función de prioridades nacionales.

\section{Tensiones en la Internacionalización de la Extensión}

En la primera década del siglo XXI se han generado importantes cambios respecto a la internacionalización universitaria en la Región, uno de ellos es la incorporación de las actividades de extensión ${ }^{6}$ como una arista de la internacionalización universitaria. Por ejemplo, en el año 2008 se incorpora la extensión en la Declaración de la CRES, entendida como: “(...) un trabajo de extensión que enriquezca la formación, colabore en detectar problemas para la agenda de investigación y cree espacios de acción conjunta con distintos actores sociales, especialmente los más postergados”. Además, en la Región se destacan diferentes instrumentos entre ellos: Unión Latinoamericana de Extensión; Congresos de Extensión de AUGM (desde 2010); Comisión permanente de extensión creada por AUGM; Jornadas Anuales de Extensión MERCOSUR; etc.

En el caso de Argentina, el Consejo Interuniversitario Nacional (CIN) acordó en el plenario no 8012/12 el 26 de marzo de 2012 en su Art. 1': "Promover la inclusión de la dimensión Extensión en las políticas de internacionalización de la Educación Superior en las Instituciones Universitarias Públicas”.

Si bien el tema de la internacionalización de la extensión no ha sido suficientemente abordado desde los estudios académicos, a partir de estudios de caso podemos identificar algunas de las tensiones que se reflejan en el ámbito interno a las universidades, vinculadas con diferentes formas de entender los vínculos de la Universidad con el entorno, por ejemplo se identifican tensiones entre la extensión entendida como la venta de "servicios" a terceros, donde prima la racionalidad económica, en línea con la internacionalización hegemónica, basada en proyectos unidireccionales, y en base a la mercantilización (LAGER, 2010), respecto a la proyección solidaria, en base a proyectos interactivos, donde se entiende a la internacionalización como el "conjunto de actividades de comunicación de los actores

\footnotetext{
${ }_{6}^{5}$ Entrevistas realizadas a investigadores de la UNLP en el marco de la tesis doctoral (OREGIONI; 2015).

${ }^{6}$ Téngase en cuenta que las actividades de extensión caracterizaron a las universidades latinoamericana desde la Reforma de Córdoba del año1918. 
universitarios con otros actores en procesos interactivos, donde cada actor aporta sus saberes y todos aprenden, contribuyendo al uso social del conocimiento con prioridad a los actores más postergados" (UNR, 2012) En ambos extremos podemos identificar numerosos ejemplos, dado que existen aspectos intermedios donde los actores se vinculan en la generación de capacidades, donde se destaca tanto la extensión como la transferencia de conocimiento por parte de las universidades a la sociedad (HERRERA, et. al., 2009)

\section{CUESTIONAMIENTOS Y REINTERPRETACIONES SOBRE LA INTERNACIONALIZIACIÓN UNIVERSITARIA EN AMÉRICA LATINA}

Al ingresar al estudio de la dinámica que adquiere la internacionalización en las diferentes funciones de la vida universitaria, se destaca la presencia de agentes, actores, percepciones e intereses que inciden en la orientación de la internacionalización, generando tensiones entre las formas de abordarla. Dichas tensiones dan cuenta del carácter político y no neutral del fenómeno, y de la incidencia de las relaciones de poder a nivel internacional, en su orientación. A modo de síntesis se destacan las principales críticas al concepto de internacionalización hegemónico, y a continuación se presentan las potencialidades de pensar en la internacionalización desde una perspectiva contra-hegemónica, entre ellas:

a) El pensamiento hegemónico se fortalece a partir de desplazar lo Nacional y Regional hacia lo global, que se presenta como "bien público global", pero se analiza al margen de las relaciones de poder que se han dado históricamente en el contexto internacional.

b) No se cuestionan las relaciones de poder entre centros y periferias del conocimiento;

c) El conocimiento se plantea como "universal" y "neutral" a las sociedades de origen;

d) Se analiza la internacionalización en términos cuantitativos, sin profundizar en los elementos cualitativos que sostienen o no los vínculos internacionales;

e) Forma parte de un discurso tecnocrático, que presenta al conocimiento en términos de atraso y/o modernidad;

f) Hace referencia a la transnacionalización de la ES, que se encuentra directamente ligada a fines mercantiles, y presenta a la educación como un servicio;

g) Genera tensiones entre los aspectos socio-cognitivos y político-institucionales en la internacionalización universitaria. (Ej. Estudios de caso UNLP; UNICEN)

h) Los parámetros de éxito se basan en rankings internacionales, donde las universidades son comparables independientemente del contexto en el que se desarrollan.

Consecuentemente, con el objetivo de transitar hacia una internacionalización nohegemónica, se plantea la importancia de proyectar la internacionalización hacia la integración de las universidades latinoamericanas desde una perspectiva solidaria y endógena, a partir de diferentes instrumentos como la cooperación académica, incluir en la curricula la creación de redes temáticas, multidisciplinarias, y asociativas de universidades destinadas a desarrollar investigación científica pertinente a las problemáticas regionales.

\begin{tabular}{l|l|l|l|l|l|l} 
(C) Rev. Inter. Educ. Sup. & Campinas, SP & v.3 & n.1 & p.114-133 & jan./abr. 2017 & ISSN 2446-9424 \\
\hline
\end{tabular}


Como sostiene Rojas Mix (2005) formar a las nuevas generaciones en concepciones mucho más amplias que abarquen e integren el conocimiento sobre la historia, la literatura, la cultura, las ciencias, y las artes en estructuras comprensivas de todo el continente latinoamericano. En este sentido, la universidad debe pensar la internacionalización en términos latinoamericanos, diferenciando la universidad para el desarrollo de la universidad para el mercado. Contemplando un denominador común en las universidades de la Región latinoamericana, que consiste en generar conocimiento relevante a las necesidades sociales (DAGNINO, 2007).

Las Redes como articuladoras de las diferentes dimensiones Universidad en función de la Internacionalización Integral

En la primera parte del artículo se describió la dinámica de la internacionalización en las diferentes dimensiones de la vida universitaria, y las tensiones que manifiestan. En la segunda parte, se propone pensar a las redes de producción y difusión de conocimiento como un instrumento que vincula los distintos aspectos de la internacionalización universitaria en función de un proyecto en concreto, en este sentido, se presentan como un instrumento de política que permite ampliar los vínculos internacionales desde una perspectiva no hegemónica, endógena e integral. Si bien se promueven desde el ámbito de la gestión, no es un instrumento línea. Consecuentemente, las redes actúan como estructuras que permiten construir lazos de cooperación entre académicos de la Región en función de problemas, donde intervienen actividades de investigación, docencia, extensión y gestión, es decir que se plantean soluciones desde una perspectiva integral. Asimismo, las redes de producción y difusión de conocimiento, posibilitan el intercambio de flujos de conocimiento a partir de la interacción de docentes, estudiantes, investigadores y gestores mediante actividades de movilidad física y virtual (OREGIONI; PIÑERO, 2014).

La universidad es el ámbito apropiado para la internacionalización integral, orientada a la resolución de problemas desde una perspectiva no-hegemónica. Como plantea Santos (2008) “(...) el único modo eficaz y emancipador de enfrentar la globalización neoliberal es contraponerle una globalización alternativa, una globalización contra hegemónica..." (2008, p.169). En este marco, el papel de la Universidad es preponderante y presenta desafíos que deben atenderse para pensar modos posibles de contrarrestar las consecuencias neoliberales:

La nueva transnacionalización alternativa y solidaria se apoya ahora en las tecnologías de la información y la comunicación, y en la constitución de redes nacionales y globales donde circulen las nuevas pedagogías, nuevos procesos de construcción y de difusión de conocimientos científicos y otros, nuevos compromisos sociales, locales, nacionales y globales. El objetivo consiste en recuperar el papel de la universidad pública en la definición y resolución colectiva de los problemas sociales, que ahora, aunque sean locales o nacionales, no se resuelven sin considerar su contextualización global. El nuevo contrato universitario parte así de la premisa de que la universidad tiene un papel crucial en la construcción del lugar del país en un mundo polarizado entre globalizaciones contradictorias. (SANTOS, 2008, p. 170).

v.3

n.1


La confluencia de distintos aspectos de la vida universitaria, da forma a la internacionalización universitaria integral, mediante redes de producción y difusión de conocimientos. En este sentido, tal como se argumenta en Oregioni y Piñero $(2015,58)$ las redes constituyen una forma de generar conocimiento en forma integral, en función de proyectos concretos, vinculados a problemas socio-cognitivos, donde se presenta: 1) la cooperación por sobre la competencia; 2) el reconocimiento de distintos tipos de pares; 3) proyección integral de la universidad en función del desarrollo endógeno de la Región: 4) privilegiando la legitimidad por sobre la competencia; 5) respeto a la diversidad regional; 6) incremento de vínculos entre docentes, investigadores, estudiantes de la Región, mediante la movilidad fisica y virtual. Particularmente se destaca el anclaje territorial de la universidad al trabajar en relación con otro tipo de pares no academicos, que presentan otro tipo de saberes, en función de la resolución de problemas.

Las políticas de internacionalización universitaria y las redes de producción y difusión de conocimiento, no funcionan en un ámbito abstracto, sino en un contexto socio-político concreto. Desde el Sector Educativo del Mercosur, se ha planteado el desafío de construir redes que permitan redefinir las prioridades, problemáticas y escenarios de las universidades públicas del Mercosur por fuera de los parámetros hegemónicos internacionales. En este sentido se destaca el trabajo de redes de producción de conocimiento vinculadas a problemáticas sociales, como es el caso de la red de estudios sobre la evaluación de la extensión (ALMADA, et. al; 2014); y que favorecen la integración Regional, como es el caso de la red del español como lengua segunda y extranjera (DANDREA, 2014). Ambos casos, se reflejan estrategias de internacionalización universitaria, endógenas y solidarias, y su creación responde a estímulos originados mediante instrumentos de política regional. ${ }^{7}$

Sin embargo, con el retorno del neoliberalismo en varios países de la Región ${ }^{8}$, se corre el riesgo de que las tensiones respecto a la orientación de la internacionalización se profundicen, incrementando la pérdida de poder de control, de autonomía y generación de estrategias endógenas por parte de las universidades, respecto a sus funciones sustantivas (investigación, docencia y extensión), que (tal como sucedió anteriormente en el contexto de políticas neoliberales) quedan subordinadas a regulaciones de organismos financieros internacionales (OFI), y consecuentemente orientada exógenamente, ya que los proveedores externos y la racionalidad económica vuelven a ocupar el centro de la escena. Tal como mencionaba Sebastián (2004), los OFI son agentes de internacionalización de la universidad, a partir de la promoción de actividades de cooperación que traen aparejadas agendas de trabajo en ocasiones cerradas y sin márgenes de negociación. "Los programas se elaboran y

\footnotetext{
${ }^{7}$ Ver Convocatorias del Núcleo de Estudios e Investigaciones del SEM.

<http://nemercosur.siu.edu.ar/index.html >

8 Luego de un periodo dónde confluyeron gobiernos progresistas, a partir de los golpes blandos en Paraguay (2012) y Brasil (2016), y el cambio de gobierno en Argentina (2015), se retoman políticas neoliberales y dependentistas, que prioriza las relaciones internacionales asimétricas, por sobre los vínculos con los países de la Región latinoamericana.

(C) Rev. Inter. Educ. Sup.

Campinas, SP

v.3

n. 1

p.114-133

jan./abr. 2017

ISSN 2446-9424
} 
deciden en las organizaciones desde sus propias lógicas y prioridades y posteriormente se implementan a través de una oferta de financiamiento" (SEBASTIÁN, 2004, p. 77).

Consecuentemente, en este contexto, se incrementa la relevancia de generar redes regionales, que pugnen por preservar el sentido de la educación como un derecho, y bregar por los efectos positivos de la internacionalización solidaria y endógena, destacando la importancia de construcciones de lazos en la producción y transmisión de conocimientos entre investigadores, docentes, estudiantes, e instituciones de diferentes países, respetando la diversidad y particularidad de las universidades de la Región latinoamericana. En este sentido, se destaca el origen de la AUGM, como respuesta a la integración neoliberal.

(...) la Asociación de Universidades del Grupo Montevideo (AUGM) ha sido un proyecto contra-hegemónico e innovador para su época, ya que se creó en el año 1991, en un momento en el que imperaba el modelo neoliberal en la región, y el mandato de los organismos financieros internacionales que orientaban la internacionalización de la universidad hacia la lógica del mercado. (OREGIONI, 2013, p. 56)

El desafío consiste en que las universidades comiencen a pensarse en relación a las sociedades a las que pertenecen, consecuentemente el anclaje territorial y la proyección de la internacionalización hacia la integración regional, cobran especial relevancia.

\section{REFLEXIONES FINALES}

De acuerdo a lo planteado, el concepto de internacionalización universitaria comienza a ganar claridad, dejando atrás las prácticas de vinculación internacional individuales basadas en actividades puntuales, para pensarlo en toda su complejidad, a partir de identificar diferentes niveles de análisis (internacional, regional, nacional, institucional), y la intervención de diferentes actores (ALBATCH, 2004) y agentes de internacionalización (SEBASTIÁN; 2011) que contribuyen y pugnan en su orientación, en un contexto internacional caracterizado por la globalización económica y la disputa geopolítica. En este sentido, el trabajo aporta elementos para entender la internacionalización universitaria desde una perspectiva transversal e integral a la funciones de sustantivas de la vida universitaria, desde una perspectiva situada en la Región latinoamericana, contemplando la incidencia de las relaciones de poder que históricamente han dado un rol marginal a la Región, y enfatizando en los desafíos que implica la globalización en el contexto actual.

A partir del análisis holístico, como reflexión final, se destacan dos elementos que es necesario tener en cuenta al momento de analizar y proyectar la internacionalización universitaria, estos son: la complejidad del fenómeno, y su carácter no neutral. La complejidad, está relacionada con su carácter transversal, dado que abarca las funciones sustantivas de la vida universitária, y consecuentemente se orienta a partir de diferentes lógicas institucionales, disciplinares, políticas, e ideológicas. Asimismo, en dicha orientación 
intervienen incentivos directos o indirectos, como: parámetros de evaluación, programas nacionales, regionales e internacionales. Por otro lado, no es neutral al proyecto de universidad, de país y de Región que se busca proyectar, consecuentemente las tensiones en su construcción tienen que ver con su carácter político. Dichas características deben contemplarse desde la política y gestión universitaria al momento de planificar la internacionalización desde una perspectiva endógena a la universidad, al país y a la Región en la que se encuentra inserta. Teniendo en cuenta 'para qué', 'cómo' y 'con qué recursos' se va abordar este proceso.

Por último, se destaca a las Redes de producción y difusión de conocimiento como un instrumento que permite potenciar la internacionalización solidaria y endógena en función de generar conocimiento pertinente a las problemáticas regionales, contribuyendo al desarrollo de la internacionalización contra-hegemónica.

\section{REFERENCIAS}

ALMADA, Julieta et al. Extensión, investigación y gestión: desafíos en la construcción de vínculos regionales. Integración y Conocimiento: Revista del Núcleo de Estudios e Investigaciones en Educación Superior del MERCOSUR, Córdoba, n.2, p.214-223, 2014. Disponible en: <https://revistas.unc.edu.ar/index.php/integracionyconocimiento/article/ view/9378>. Acceso en: 10 sep. 2016.

ALTBACH, Philip. Globalization and the university: myths and realities in an unequal World. Tertiary Education and Manangement, London, n. 10, p. 3-25, 2004.

BERNAL, Marcelo. La internacionalización de la Educación Superior: el debate actual de los años noventa hasta el presente, y sus futuras implicancias. Córdoba: Editorial de la Universidad Católica de Córdoba, 2007.

CONFERENCIA REGIONAL DE LA EDUCACIÓN SUPERIOR EN AMÉRICA LATINA Y EL CARIBE (CRES). Declaraciones y plan de acción. Perfiles educativos, Buenos Aires, v.31, n.125, p.90-108, 2009. ISSN 0185-2698.

DAGNINO, Renato. A comunidade de pesquisa ea política de ciência e tecnologia: olhando para os países avançados. Revista Iberoamericana de Ciencia Tecnología y Sociedad, Buenos Aires, v.3, n.7, p. 43-58, 2006.

DAGNINO, Renato. La universidad y el desarrollo de América Latina. Atos de Pesquisa em Educação - Ppge/Me Furb, v.2, n.3, p. 371 -382, 2007.

DANDREA Fabio. Articulación Lingüístico Cultural para una Cooperación Interinstitucional Solidaria. Proyecto de Red NEIES: Integración Regional para ELE en el contexto MERCOSUR. Integración y Conocimiento: Revista del Núcleo de Estudios e Investigaciones en Educación Superior del MERCOSUR, Córdoba, n.2, 2014. Disponible en: 
$\langle$ https://revistas.unc.edu.ar/index.php/ integracionyconocimiento/article/view/9381 $>$. Acceso en: 10 sep. 2016

DIDOU AUPETIT, Silvie. La internacionalización de la educación superior en América Latina: oportunidades y desafíos. En: CONFERENCIA DICTADA EN EL PABELLÓN ARGENTINA DE LA CIUDAD UNIVERSITARIA, 2007, Córdoba. Anales... Córdoba: [s.n.], 2007.

DIDOU AUPETIT, Sylvie; JARAMILLO DE ESCOBAR, Vielka. Internacionalización de la Educación Superior y la Ciencia en América Latina: un estado del arte.[S.1.]: IESALCUNESCO, 2014.

FERNANDEZ LAMARRA, Norberto. La Convergencia de la Educación Superior: hacia la creación de un espacio común latino-americano - maneiro. Integración, cooperación e Internacionalización de la Educación Superior, p. 133-168, 2009.

GARCÍA DE FANELLI, Ana María. La educación transnacional: la experiencia extranjera y lecciones para el diseño de una política de regulación en la Argentina. Buenos Aires, CONEAU, 1999.

GASCÓN MURO, Pablo; CEPEDA DOVALA, Jesús. De la mercantilización a la transnacionalización de la educación superior. Revista Reencuentro. Análisis de problemas universitarios, México, no 40, ago. 2004.

HERRERA, Alma et. al. La responsabilidad social de las macro-universidades de América Latina y el Caribe. Universidades, v.59, n.41, p.11-26, abr./jun. 2009 Disponible en: $<$ http://redalyc.uaemex.mx /src/inicio/ArtPdfRed.jsp?iCve=37313031004> Acceso en: 16 fev. 2016

HODARA, Joseph. Ciencia en la periferia de la periferia: hacia la formación de colegios virtuales. Estudios Interdisciplinarios de América Latina y El Caribe, 2003.

KNIGHT, Jane. Internationalization: elements and checkpoints. Otawa: Oficina de Canadá para la Cooperación Internacional, 1994. (Monografía Investigativa; n.7)

KNIGHT, Jane. Internationalization of higher education. En: KNIGHT, J.; WIT, H. de (Ed.). Quality and internationalization in higher education. Paris: IHME/OECD,1999.

KREIMER, Pablo; MEYER, J. B. Equality in the networks? some are more equal than others: international scientific cooperation: an approach from Latin America. En: VESSURI, H.; TEICHLER, U. Universities as centers of research and knowledge creation: an endangered species? Rotterdam: Sense Publishers, 2008.

KREIMER, Pablo; THOMAS, Hernán. Production des connaissances dans la science périphérique: l' hypothèse CANA en Argentine. En: MEYER, J. B.; CARTON, M. La société des saviors: trompe l'œil ou perspectives? París: [s.n.], 2006. p. 143-169.

KREIMER, Pablo; ZABALA, Juan Pablo. ¿Qué conocimiento y para quién? problemas sociales, producción y uso social de conocimientos científicos sobre la enfermedad de Chagas en Argentina. Revista Redes, Buenos Aires, v.12, n.23, p.49-78, 2006.

\begin{tabular}{l|l|l|l|l|l|l} 
(C) Rev. Inter. Educ. Sup. & Campinas, SP & v.3 & n.1 & p.114-133 & jan./abr. 2017 & ISSN 2446-9424 \\
\hline
\end{tabular}


LANGER, Ariel. Investigación, transferencia, extensión y docencia: análisis de las concepciones en la política científica y universitaria de la Argentina, 2010. Disponible en: <http://www.anped11.uerj.br/28/Langer\%20Ariel.doc $>$. Acceso en: 23 sep. 2013.

NAIDORF, Judith. Relaciones académicas internacionales. Formas de enriquecer las relaciones bilaterales. En: SEMINARIO INTERUNIVERSITARIO DE ESTUDIOS CANADIENSES EN AMÉRICA LATINA, 3.: 2005, La Habana. Anales...La Habana: [s.n.], 2005.

OREGIONI, María S.; PIÑERO Fernando. Redes de producción y difusión de conocimiento ¿un instrumento para orientar la internacionalización de la Universidad Argentina hacia América Latina? En: ARAYA, José Maria J. (Comp.). Aportes para los estudios sobre internacionalización de la educación superior en América del Sur. Buenos Aires: UNICEN, 2015. p. 51-57.

OREGIONI, María Soledad. ¿De qué hablamos cuando hablamos de internacionalización universitaria? En: LAGO MARTÍNEZ, Silvia; CORREA, Néstor Horacio. (Coord.). Desafíos y dilemas de la universidad y la ciencia en América Latina y el Caribe en el siglo XXI. Buenos Aires: Editorial Tasseo Pres, 2015. Disponible en: <https://www.teseopress.com/ universidad yciencia/chapter/de-que-hablamos-cuando hablamos-de-internacionalizacionuniversitaria-2/>. Acceso en: 10 oct. 2016

OREGIONI, María Soledad. Aspectos Sociales de la Internacionalización de la Investigación, una propuesta de abordaje. Revista Iberoamericana en Ciencia, Tecnología y Sociedad, Buenos Aires, v.10, n.30, p.199-229, sep. 2014. Disponible en:

$<$ http://www.revistacts.net/volumen-10-numero-30/307-articulos/693-aspectos-sociales-dela-internacionalizacion-de-la-investigacion-una-propuesta-de-abordaje >. Acceso: 23 sep. 2016

OREGIONI, María Soledad. Dinámica de la Internacionalización de la Investigación en la Universidad Nacional de La Plata. 2014. Tesis (Doctorado en Ciencias Sociales y Humanas) - Universidad Nacional de Quilmes, 2014.

OREGIONI, María Soledad. Incidencia directa e indirecta de la Internacionalización de la Investigación en la Universidad Nacional de La Plata, desde una perspectiva multidimensional (en línea). En: JORNADAS DE SOCIOLOGÍA DE LA UNLP, 8.: 2014, Ensenada, Argentina. Anales... Ensenada, Argentina: UNPL, 2014. Disponible en: $<$ http://www.memoria.fahce.unlp.edu.ar/trab_eventos/ev.4311/ev.4311.pdf $>$ Acceso: 21/09/2016.

OREGIONI, María Soledad. La universidad como actor de la cooperación sur- sur: el caso de la UNLP en la Asociación de Universidades del Grupo Montevideo. Integración y Conocimiento: Revista del Núcleo de Estudios e Investigaciones en Educación Superior del MERCOSUR n.2, 2013. Disponible en: <https://revistas.unc.edu.ar/index.php/integracion yconocimiento/article/view/5893>. Acceso en: 23 ago. 2016

OREGIONI, María Soledad et. al. Proyección Integral de la Universidad Argentina hacia la Región Latinoamericana: agenda, actores e instrumentos. En: JORNADAS DE EXTENSIÓN 
DEL MERCOSUR. UNCPBA, 2014. Anales... Disponible en: <http://extension.unicen. edu.ar/jem/completas/340.pdf $>$. Acceso en: 10 oct. 2016.

PELLEGRINO, Adela. Drenaje, movilidad, circulación: nuevas modalidades de la migración calificada. En: SIMPOSIO SOBRE MIGRACIÓN EN LAS AMÉRICAS. Actas del..., San José de Costa Rica: CEPAL- OIM, 2001. p. 129-162.

PERROTTA, Daniela. La Región Sudamericana como arena política para la internacionalización de la universidad. En: ARAYA, José Maria J. (Comp.). Aportes para los estudios sobre internacionalización de la educación superior en América del Sur. Buenos Aires: UNICEN, 2015. p.21-50.

ROJAS MIX, Manuel. Siete preguntas sobre la Universidad Latinoamericana del siglo XXI. En: EDUCACIÓN superior ¿bien público o bien de mercado? Buenos Aires: Ministerio de Educación, Ciencia y Tecnología, 2005.

SANTOS, Boaventura de Sousa. El rol de la Universidad en la construcción de una globalización alternativa. En: LA EDUCACIÓN superior en el mundo 2008: la financiación de las universidades. Catalynia, España: Editorial Mundiprensa, 2008. ISBN 978-84-8476-343-7. Disponible en: <http://upcommons.upc.edu/handle/2099/7981 >. Acceso en: 10 oct. 2016.

SEBASTIÁN, Jesús. Dimensiones y métrica de la internacionalización de las universidades. Universidades, Mexico, n.61, oct./dic. 2011. ISSN 0041-8935 Disponible en: <http://www.redalyc.org/articulo.oa?id=37322089002>. Acceso en: 10 oct. 2016.

SEBASTIÁN, Jesús. Conocimiento, cooperación y desarrollo. Revista Iberoamericana de Ciencia, Tecnología y Sociedad, Buenos Aires, v.3, n.8, p.195-208, 2007.

SEBASTIÁN, Jesús. Cooperación e internacionalización de la universidades. Buenos Aires: Biblos, 2004.

SIUFI, Gabriela. Cooperación internacional e internacionalización de la educación superior. Venezuela: UNESCO-IESALC, 2009.

VESSURI, Hebe. El papel cambiante de la investigación científica académica en un país periférico. En: DÍAZ, E. et al. (Ed.). La ciencia periférica: ciencia y sociedad en Venezuela. Caracas: Monte Ávila Editores, 1984. p.37-72.

VESSURI, Hebe. Scientific Cooperation among Unequal partners: the strait-jacket of the human resource base. En: GAILLARD, J. (Ed.). Coopérations scientifiques internationals: les sciences hors d'occident au xxe siècle. París: ORSTOM, 1996. p.171-185.

YARZÁBAL, Luis. Internacionalización de la educación superior: de la cooperación académica al comercio de servicios. En: PUGLIESE, J. (Ed.). Educación superior ; bien público o bien de mercado? los debates sobre la Internacionalización desde los noventa a la actualidad. Buenos Aires: MECyT-SPU, 2005. 